Hadron 90 is that this seems to be the case, at least for the $0^{-}$quantum number ('pseudoscalar states'). This begins to add empirical encouragement to many theorists who have argued (the most recent in a long line being G. J. Gounaris and J. E. Paschalis; Phys. Lett. B251, 634-638; 1990) that detailed structures in spectroscopic data around $1.4 \mathrm{GeV}$ mass are best understood if there is a 0 glueball.

The importance in identifying a detailed spectroscopy of glueballs is that sighting bound states of gauge fields (such as glueballs), as distinct from those of fermions (the conventional 'material' particles), would be entirely new. We have good evidence that the underlying mathematical structure of QCD and QED are very similar but the richness of the SU(3) non-Abelian structure of QCD implies that there are novel possibilities such as non-trivial vacua, instantons and probably further profound consequences that will be revealed only through experiment. To identify these it is important to isolate the spectroscopy of the glueballs. Theory suggests that glueballs are accessible at rather low energies, well within the reach of existing laboratories. It will be the advances in detector technology and particle beam quality and intensity that will be the keys to opening up this area of particle physics, and will offer the hope of deepening insights into the physics of nonAbelian gauge field theories.

Frank Close is at the Rutherford-Appleton Laboratories, Chilton, Didcot $\mathrm{OX} 11 \mathrm{OQH}$, UK, and Oak Ridge National Laboratory, PO Box 2008, Oak Ridge, Tennessee 37831, USA.

\title{
Real and imagined dangers
}

\section{Gordon Ada}

EARLIER this month a report by Oehen et al. appeared in Science ${ }^{1}$, under the title "Vaccination for Disease", in which the authors describe some experiments with lymphocytic choriomeningitis virus (LCMV) in mice. This virus contains two main protein types, glycoproteins and a nucleoprotein. In some experiments, preimmunization of mice with vaccinia virusLCMV single protein constructs exacerbated the disease that occurred when the mice were challenged with LCMV administered intracerebrally. In contrast, preimmunization with intact LCMV protected the mice. Although these experiments are valid and helpful lessons can be drawn from them, the very title of the paper might be inappropriately taken as a cause for alarm, particularly as chimaeric viruses (or bacteria) have already been successfully used for vaccination.

Elsewhere, I have proposed ${ }^{2}$ that a vaccine should meet four immunological requirements, three of which are relevant here. One is that the vaccine should contain a sufficient number of different T-cell epitopes that $\mathrm{T}$-cell responses will be achieved in all members of a genetically diverse outbred population. Inclusion of several different proteins in the preparation increases the number of available epitopes. A second requirement is that the vaccine should generate a large pool of memory $\mathrm{T}$ (and $\mathrm{B}$ ) cells; the greater the size of this T-cell pool, the quicker the appearance of effector $\mathrm{T}$-cell activity generated from the memory cells by the challenge virus. The results of Oehen et al. are consistent with these points, as shown in their Figs 1 and $2 \mathrm{C}$. As expected, intact LCMV which contains all the viral antigens was more protective than either of the chimaeric vaccinia virus constructs.
A third requirement of a vaccine is the generation and persistence of high titres of neutralizing antibody so that the great bulk of a challenge virus, at any time after immunization, is prevented from infecting susceptible cells. Oehen et al. did not measure such antibody generated by any of the immunization schedules, but it is very likely that immunization with whole virus would have achieved this most effectively. This, as well as the rapid appearance of effector $T$ cells after challenge (their Fig. 2C), may have been a deciding factor in preventing the deaths of animals that had been preimmunized with intact virus.

Several, more general, points should also be made. First, it is inevitable that $T$-cell activation will result in some immunopathological effects. Interleukins, which are defined as mediators of inflammation, are secreted by both $\mathrm{CD}^{+}$and $\mathrm{CD}^{+}$cells. The trick is to limit the effect as far as possible and this can be done by minimizing the extent of infection that occurs upon challenge. The risk of immunopathology becomes greater when, for reasons such as great antigenic variation, the virus largely escapes the neutralization of infectivity by preformed antibody.

Second, LCMV infection is an unusual model. Challenge by intracerebral injection, used in the experiments of Oehen $e t$ $a l$, is an unnatural route of infection. Virus inoculated into the brain can form a reservoir not easily available to the immune system. Other than vector-borne agents, most agents infect through a mucosal surface and there is increasing interest in developing vaccines which will stimulate a secretory $\operatorname{Ig} \mathrm{A}$ response as this is the first line of defence.

Third, there are many examples with other viruses where transfer of virusspecific cytotoxic $T$ cells before or after administration of a lethal dose of virus has cleared the infection and prevented death. In addition, there are many examples in model systems where immunization with a chimaeric virus preparation has provided protection against a challenge dose of the infectious agent which was the source of the foreign antigen $(\mathrm{s})^{3}$. Two outstanding examples are vaccinia virus constructs which contain antigen from rabies virus ${ }^{\text {t.5 }}$ or from rinderpest virus ${ }^{6}$ - foxes immunized with the vaccinia-rabies virus construct survived challenge with many thousands of lethal doses of rabies virus; cattle immunized with the vacciniarinderpest virus construct were protected against challenge by a thousand lethal doses of rinderpest virus.

Most vaccines currently in use are to control acute infections. Among the most successful are those consisting of live, attenuated viruses which induce cytoxic T-cell responses. Many of the infectious agents that are the target for vaccine development cause chronic persisting infections. The observations of Oehen et al. may be relevant in some of these cases, for example in HIV infections where there is great antigenic variation in the envelope protein. A candidate vaccine now in phase 1 human trials is a construct composed of vaccinia virus and the HIV envelope protein. The rationale behind this approach is to generate cytotoxic $\mathrm{T}$-cell responses which would limit infection caused by either free virus or infected cells. It remains highly desirable that such a vaccine should still aim to induce cross-protective neutralizing antibodies to limit the extent of viral infection upon initial exposure, and this may now be possible? The current development of a vaccinia virus construct containing three HIV antigens is a useful further approach to achieve rapid elimination of infected cells.

Although it may never be possible to guarantee that a given approach will always result in a completely safe vaccine, enough is now known about the important immunological requirements for vaccines, especially for viral infections, to proceed with confidence in developing the next generation. The use of live vectors, viruses or bacteria, is one of several approaches in use; it may turn out to be the best in some situations.

Gordon Ada is in the School of Hygiene and Public Health. The Johns Hopkins University, Baltimore, Maryland 21205, USA.

\footnotetext{
1. Oehen, S., Hengartner, H. \& Zinkernagel, R.M. Science 251, 195-198 (1991)

2. Ada, G. L. Lancet 335, 523-526 (1990)

3. Flexner, C. \& Moss, B. in New Generation Vaccines (eds Woodrow, G.C. \& Levine, M.M.) 189-206 (Dekker, New Woodrow, G.

4. Blancou, F. et al. Nature 332, 373-375 (1986)

5. Brochien, B. et al. Vaccine 8, 101-104 (1989)

6. Yilma, T.D. et al. Science 242, 1058-1061 (1988)

7. Javaherian, K. et al. Science 250, 1590-1593 (1990)
} 